\title{
Studies on Magnitude of Genetic Variability and Estimates of Combining Ability for Yield and its Contributing Traits among Improved Lines of Castor (Ricinus communis L.)
}

\author{
P. Bindu Priya ${ }^{1 *}$, M.V. Nagesh Kumar ${ }^{2}$, V. Gouri Shankar ${ }^{3}$, G. Seshu ${ }^{1}$, \\ G. Anuradha ${ }^{1}$ and M. Venkata Ramana ${ }^{1}$ \\ ${ }^{1}$ Regional Agricultural Research Station, Palem-509215, PJTSAU, India \\ ${ }^{2}$ Seed Research and Technology Centre, PJTSAU, Rajendranagar, India \\ ${ }^{3}$ College of Agriculture, PJTSAU, Rajendranagar, India \\ *Corresponding author
}

\begin{tabular}{|c|}
\hline Keywords \\
\hline $\begin{array}{l}\text { Castor, Combining } \\
\text { ability, Variability, } \\
\text { Heritability, } \\
\text { Selection, Crop } \\
\text { improvement }\end{array}$ \\
\hline Article Info \\
\hline $\begin{array}{l}\text { Accepted: } \\
26 \text { December } 2017 \\
\text { Available Online: } \\
\text { 10 January } 2018\end{array}$ \\
\hline
\end{tabular}

\section{Introduction}

Castor (Ricinus communis L.) plays an important role in the country's vegetable oil economy. The crop is grown for its non-edible oil (45-50 per cent oil in seeds) which is

\section{A B S T R A C T}

Thirty castor hybrids were developed by crossing three pistillate lines with ten male parents in a line $\times$ tester mating design during rabi, 2016-17. The hybrids along parents were evaluated during kharif, 2017-18 for variability and combining ability. The analysis of variance for nine economically important characters indicated significant differences among the genotypes, implying presence of wide diversity in the material studied. High heritability (broad sense) estimates were observed for all the traits under study which showed that the variability in phenotypic expression reflects the genotypic ability to transmit the genes to their offspring. The character seed yield recorded high heritability coupled with high genetic advance as per cent of mean signifying that improvement of seed yield can be achieved by adopting simple selection procedures through which additive genes can be pyramided. Relative magnitude of general and specific combining ability variances indicated that additive genetic action was predominant in governing the traits days to maturity and 100 seed weight. Non-additive gene action was predominant for the traits days to 50 per cent flowering, number of nodes up to primary spike, plant height, primary spike length, number of spikes per plant, seed yield per plant and oil content. Four lines viz., DPC-9, PCS-124, SKI-215 and JC-12 were observed to be good combiners as indicated by their significant positive gca effects for many characters. Four hybrid combinations viz., DPC-9 x PCS-124, DPC-9 x SKI-215, DPC-9 x PCS-106 and DPC-9 x JC-12 were identified as promising based on their $s c a$ estimates and per se performance. 
manure. In 2015-16, castor occupied an area of 10.60 lakh ha with a production of 17.51 lakh tons showing a significant increase in productivity levels (1652 $\mathrm{kg} / \mathrm{ha})$ which is highly encouraging. Besides meeting the domestic requirements towards its use in various fields and a variety of diversified products the country is presently dominating international castor oil market.

Diversity is the most crucial feature of any population as presence of variability is a prerequisite to entail response to selection in any crop improvement programme. Selection for superior genotypes is possible only when adequate variability exists in the gene pool. Hence, an insight into the magnitude of variability present in the gene pool of a crop species is of utmost importance to a plant breeder for initiating a judicious plant breeding programme. The coefficients of variation expressed at phenotypic and genotypic levels are generally used for comparing variability present for different characters. Hence, knowledge about the variability parameters like genotypic coefficient of variation (GCV) and phenotypic coefficient of variation (PCV) is of paramount importance for planning a proficient breeding programme. It is highly difficult to identify a genotype exhibiting superior performance for all the characters implied, hence it is prudent to target improvement of individual traits in the order of their economic importance and requirement. The heritability estimates aid in determining the relative amount of heritable portion in variation thus directing towards selection of elite genotypes from a diverse population. Therefore, the present study was undertaken for assessing the extent of genetic variability, heritability and genetic advance in castor.

Seed yield, a complex metric trait, is an ultimate product of action and interaction of a number of component characters. Further improvement in yield of this important oilseed species demands information regarding the nature of combining abilities among a wide array of genetic materials to be used as parents in the hybridization programme, as well as the nature of gene action involved in the expression of quantitative traits of economic importance. Combining ability is most helpful in determining the appropriate parents of a cross thereby comprehending suitable breeding approach for handling a cross to develop a cultivar.

\section{Materials and Methods}

Three elite pistillate lines (DPC-9, M-574 and PPL-18) were crossed with ten diverse male parents (PCS-202, PCS-225, PCS-124, SKI215, PCS-223, JC-12, PCS-136, PCS-106, PCS-171 and DCS-78) during rabi 2016-17. The resultant thirty hybrids were evaluated along with their parents and two standard checks viz., PCH-111 and GCH-4 in a randomized block design, replicated thrice at Regional Agricultural Research Station, Palem during kharif 2017-18.

The site of experimentation falls under semi arid climate and comes under the ambit of Southern Telangana Zone on $16^{0} 35^{1}$ latitude, $78^{0} 1^{1}$ longitude and altitude of $642 \mathrm{~m}$ above mean sea level. Each entry was sown in four rows with a row length of $6 \mathrm{~m}$. Ten plants were randomly selected from each entry and replication on which observations were recorded on nine economically important traits $v i z$., days to 50 per cent flowering, days to maturity, number of nodes up to primary spike, plant height $(\mathrm{cm})$, primary spike length (cm), number of spikes per plant, 100 seed weight $(\mathrm{g})$, seed yield per plant $(\mathrm{g})$ and oil content (per cent). Days to 50 per cent flowering and days to maturity were recorded on plot basis. A standard package of practices was followed for raising the crop. 
Data was analyzed using analysis for variance (Panse and Sukhatme, 1984). The estimates of PCV and GCV were classified as given by Sivasubramanian and Madhavamenon (1973). The phenotypic and genotypic co-efficient of variability were calculated as per Burton and De Vane (1953). Heritability estimates in broad sense for yield components of castor genotypes were estimated and categorized as suggested by Robinson et al., (1949), while genetic advance was worked out as per Johnson et al., (1955). Additive and dominant components of variance were also computed (Singh and Chaudhary, 1977; Dabholkar, 1992). The percentage contribution of lines, testers and their interactions were computed as per the formulae suggested by Singh and Chaudhary, 1977. Mean values were subjected to analysis using the computer programme package Windowstat 8.0 (Indostat Services, Hyderabad, India). Data was subjected to line $\mathrm{x}$ tester analysis (Kempthorne, 1957) to estimate general and specific combining ability effects and their respective variances.

\section{Results and Discussion}

The accomplishment of a breeding programme largely relies on choice of appropriate breeding methods. Heterosis studies provide information about percentage increase/decrease of $F_{1}$ over the better parent or standard checks only thus aiding in identification of the best cross but they do not indicate the possible cause for superiority of crosses. Enhancement of polygenic traits like yield demands information about the type of gene action involved while the estimates of components of variance play a vital role in formulation of crossing plans and breeding strategies.

The common approach of choosing the parents on the basis of performance, adaptation and genetic variability does not necessarily lead to productive outcomes. This is because of differential ability of the parents which depends upon the complex interactions among the genes and cannot be judged by the performance alone (Allard, 1960).

The analysis of variance (Table 2) for nine characters revealed significant differences among the genotypes, indicating wide diversity in the material studied. The range of variation was maximum for seed yield per plant (77.67-148.33 g) followed by plant height $(50.27-90.13 \mathrm{~cm})$ and days to 50 per cent flowering (37.00-59.67 days). Higher mean was associated with high range, indicating the scope of improvement through simple selection procedures. PCV was greater than GCV for all the characters under study indicating the environmental effect (Table 1). The coefficients of variation displayed that the magnitude of phenotypic and genotypic variability was medium for the traits number of spikes per plant, plant height and seed yield per plant while low variability was observed for oil content and days to maturity. Similar findings were reported by Sood and Kumar (2011).

However, high variance values alone are not the determining factors of the expected progress that could be made in quantitative traits (Falconer, 1981). It was suggested that the GCV together with the high heritability $\left(h^{2}\right)$ estimates would give better portrait of extent of genetic gain to be expected under selection. In the present study, all the characters expressed high heritability estimates ranging from 61 to 89 per cent. High heritability (broad sense) estimates were observed for all the traits indicating that dependence of phenotypic expression reflects the genotypic ability to transmit genes to their offspring. These results are in accordance with Ramesh et al., (2012). Genetic advance as per cent of mean was highest for plant height followed by number of spikes per plant and seed yield per plant. However, the traits 
primary spike length, number of nodes per plant, days to 50 per cent flowering and 100 seed weight recorded medium genetic advance while oil content and days to maturity observed low genetic advance as per cent of mean.

High heritability with high genetic advance as per cent of mean was observed for plant height followed by number of spikes per plant and seed yield per plant. These results are in agreement with the observations made by Patel et al., (2010). Seed yield recorded high heritability coupled with high genetic advance as per cent of mean suggesting that improvement in seed yield can be achieved by adopting simple selection procedures with which additive genes can be pyramided and will be gaining in the selection process.

In a crop improvement program, much of the success depends upon isolation of valuable gene combinations as determined in the form of lines with high combining ability (Punewar et al., 2017).

A comparison of relative magnitude of general and specific combining ability variances (Table 2) indicated that additive genetic action was predominant in governing the traits days to maturity and 100 seed weight. Non-additive gene action was predominant for the traits days to 50 per cent flowering, number of nodes up to primary spike, plant height, primary spike length, number of spikes per plant, seed yield per plant and oil content (Table 3). These results are similar to the findings of Mehta (2000), Ramu et al., (2002), Lavanya et al., (2006), Solanki (2006), Chandramohan et al., (2006) and Patel et al., (2007).

Among the lines studied DPC-9 and among the testers PCS-124, SKI-215 and JC-12 were good combiners as indicated by their significant positive gca effects (Table 4). All these parents contribute maximum positive alleles for the increase of seed yield per plant and oil content. The line DPC-9 is a good combiner for days to 50 per cent flowering, number of nodes up to primary spike, number of spikes per plant, seed yield per plant and oil content (Table 4). Among the testers, PCS-124 was found to be a good general combiner for days to 50 per cent flowering, number of spikes per plant, 100 seed weight, seed yield per plant and oil content, whereas SKI-215 for number of spikes per plant, 100 seed weight, seed yield per plant and oil content, JC-12 for primary spike length, number of spikes per plant, 100 seed weight, seed yield per plant and oil content. These parents can be effusively used in breeding programmes in various cross combinations for improvement in seed yield and other agronomic characters because of their ability to transmit characters to off springs. Similar results were obtained by Solanki et al., (2004), Thakker et al., (2005) and Venkataramana et al., (2005).

Among the hybrids, four combinations viz., DPC-9 x PCS-124, DPC-9 x SKI-215, DPC-9 $x$ PCS-106 and DPC-9 x JC-12 were identified as promising based on the specific combining ability and per se performance. In each of the four crosses, both the parents were found to be good combiners (Table 4). Patel et al., (2012), Kasture et al., (2014), Aher et al., (2015) and Pawar and Sakhare (2016) also reported similar results.

When we compare per se performance with gca effects of parents or sca effects of hybrids, some crosses were common but occupied different positions. It is suggested that selection of cross combination should be made on the basis of per se performance as well as sca effects. A cross combination exhibiting high sca effects and involving parents with high gca effects is most useful in crop improvement programme. 
Table.1 Estimates of genetic components of variance

\begin{tabular}{|c|c|c|c|c|c|c|c|c|c|}
\hline Components & $\begin{array}{c}\text { Days to } \\
50 \text { per } \\
\text { cent } \\
\text { flowering }\end{array}$ & $\begin{array}{l}\text { Days to } \\
\text { maturity }\end{array}$ & $\begin{array}{l}\text { Nodes } \\
\text { up to } \\
\text { primary } \\
\text { spike }\end{array}$ & $\begin{array}{l}\text { Plant } \\
\text { height } \\
(\mathrm{cm})\end{array}$ & $\begin{array}{l}\text { Primary } \\
\text { spike } \\
\text { length } \\
(\mathrm{cm})\end{array}$ & $\begin{array}{c}\text { Number } \\
\text { of } \\
\text { spikes/ } \\
\text { plant }\end{array}$ & $\begin{array}{c}100 \\
\text { seed } \\
\text { weight } \\
(\mathrm{g})\end{array}$ & $\begin{array}{l}\text { Seed } \\
\text { yield/ } \\
\text { plant } \\
(\mathrm{g})\end{array}$ & $\begin{array}{c}\text { Oil } \\
\text { content } \\
(\%)\end{array}$ \\
\hline Range Lowest & 37 & 82.33 & 9.2 & 50.27 & 41.9 & 2.33 & 21.33 & 77.67 & 45 \\
\hline Range Highest & 59.67 & 92.33 & 15.83 & 90.13 & 66.93 & 5.27 & 31 & 148.33 & 50.07 \\
\hline$\sigma^{2} \mathbf{P}($ Phenotypic Variance) & 23.79 & 9.25 & 2.17 & 150.36 & 57.09 & 0.59 & 9.13 & 318.63 & 2.16 \\
\hline$\sigma^{2}$ G (Genotypic Variance) & 21.07 & 6.69 & 1.79 & 132.65 & 45.59 & 0.43 & 7.61 & 272.59 & 1.31 \\
\hline $\begin{array}{l}\sigma^{2} \mathrm{E}(\text { Environmental } \\
\text { Variance) }\end{array}$ & 2.73 & 2.56 & 0.38 & 17.70 & 11.50 & 0.16 & 1.52 & 46.04 & 0.85 \\
\hline PCV & 10.94 & 3.50 & 13.55 & 18.22 & 14.51 & 20.42 & 11.56 & 16.47 & 3.15 \\
\hline GCV & 10.30 & 2.97 & 12.30 & 17.11 & 12.97 & 17.41 & 10.55 & 15.24 & 2.45 \\
\hline ECV & 3.70 & 1.84 & 5.70 & 6.25 & 6.51 & 10.67 & 4.72 & 6.26 & 1.98 \\
\hline$h^{2}($ Broad Sense $)$ & 0.89 & 0.72 & 0.82 & 0.88 & 0.80 & 0.73 & 0.83 & 0.86 & 0.61 \\
\hline Genetic Advancement 5\% & 8.90 & 4.53 & 2.50 & 22.29 & 12.43 & 1.15 & 5.19 & 31.46 & 1.83 \\
\hline $\begin{array}{l}\text { Gen. Adv. as per cent of } \\
\text { Mean } 5 \text { per cent }\end{array}$ & 19.96 & 5.21 & 22.99 & 33.11 & 23.87 & 30.57 & 19.85 & 29.03 & 3.93 \\
\hline
\end{tabular}


Table.2 Analysis of variance for seed yield and yield components in castor

\begin{tabular}{|c|c|c|c|c|c|c|c|c|c|c|}
\hline & df & $\begin{array}{l}\text { Days to } 50 \\
\text { per cent } \\
\text { flowering }\end{array}$ & $\begin{array}{l}\text { Days to } \\
\text { maturity }\end{array}$ & $\begin{array}{l}\text { No. of nodes } \\
\text { up to primary } \\
\text { spike }\end{array}$ & $\begin{array}{l}\text { Plant height } \\
(\mathrm{cm})\end{array}$ & $\begin{array}{c}\text { Primary } \\
\text { spike length } \\
(\mathrm{cm})\end{array}$ & $\begin{array}{l}\text { No. of } \\
\text { spikes/ } \\
\text { plant }\end{array}$ & $\begin{array}{l}100 \text { Seed } \\
\text { weight }(g)\end{array}$ & $\begin{array}{l}\text { Seed yield/ } \\
\text { plant (g) }\end{array}$ & $\begin{array}{c}\text { Oil } \\
\text { Content } \\
(\%)\end{array}$ \\
\hline Replicates & 2 & 0.30 & 2.43 & 0.03 & 1.83 & 0.60 & 0.14 & 0.78 & $158.30^{*}$ & 2.33 \\
\hline Treatments & 42 & $68.01 * *$ & $23.68 * *$ & $5.97 * *$ & $422.96 * *$ & $150.99 * *$ & $1.45^{* *}$ & $25.44 * *$ & $889.78 * *$ & $4.91 * *$ \\
\hline Parents & 12 & $12.79 * *$ & $25.86 * *$ & $8.06 * *$ & $181.14 * *$ & $35.39 * *$ & $1.42 * *$ & $19.42 * *$ & $831.08 * *$ & $2.06 *$ \\
\hline Parents (Line) & 2 & 4.78 & 3.11 & $2.33 * *$ & 49.67 & 5.79 & $0.52 *$ & 2.33 & 33.78 & 0.22 \\
\hline Parents (Testers) & 9 & $15.99 * *$ & $24.43 * *$ & $9.43 * *$ & $222.10 * *$ & $38.61 * *$ & $1.42 * *$ & $17.17 * *$ & $910.73 * *$ & $2.67 * *$ \\
\hline Parents (L vs T) & 1 & 0.01 & $84.27 * *$ & $7.20 * *$ & $75.49 *$ & $65.58 *$ & $3.24 * *$ & $73.88 * *$ & $1708.89 * *$ & 0.22 \\
\hline Parents vs Crosses & 1 & $1371.95 * *$ & $35.27 * *$ & $3.73 * *$ & $1873.37 * *$ & $2246.73 * *$ & $1.79 * *$ & $89.08 * *$ & $9315.83 * *$ & $10.77 * *$ \\
\hline Crosses & 29 & $45.90 * *$ & $22.38 * *$ & $5.18 * *$ & $473.00 * *$ & $126.57 * *$ & $1.44 * *$ & $25.74 * *$ & $623.51 * *$ & $5.89 * *$ \\
\hline Line Effect & 2 & 101.48 & 1.23 & $11.69 *$ & 785.35 & 259.72 & 1.57 & 2.01 & $1296.21 * *$ & $7.01^{*}$ \\
\hline Tester Effect & 9 & 41.26 & $64.77 * *$ & $8.76 *$ & $820.17 *$ & 88.46 & $3.31 * *$ & $76.97 * *$ & $1535.33 * *$ & $14.72 * *$ \\
\hline Line * Tester Eff. & 18 & $42.05 * *$ & 3.53 & $2.67 * *$ & $264.71 * *$ & $130.82 * *$ & $0.50 * *$ & $2.75^{*}$ & $92.85 *$ & 1.35 \\
\hline Error & 84 & 2.72 & 2.64 & 0.38 & 16.44 & 11.71 & 0.16 & 1.53 & 45.81 & 0.89 \\
\hline Total & 128 & 24.10 & 9.54 & 2.21 & 149.60 & 57.24 & 0.58 & 9.36 & 324.49 & 2.23 \\
\hline
\end{tabular}

* Significant at 5\% level, ** Significant at $1 \%$ level

Table.3 Estimates of general and specific combining variances and proportionate gene action for nine characters in castor

\begin{tabular}{|c|l|c|c|c|}
\hline S.No. & \multicolumn{1}{|c|}{ Character } & $\sigma^{2}$ gca & $\sigma^{2}$ sca & $\sigma^{2}$ gca/ $\sigma^{2}$ sca \\
\hline $\mathbf{1}$ & Days to 50 per cent flowering & 0.07 & 13.11 & 0.01 \\
\hline $\mathbf{2}$ & Days to maturity & 0.35 & 0.29 & 1.21 \\
\hline $\mathbf{3}$ & Nodes up to primary spike & 0.04 & 0.76 & 0.05 \\
\hline $\mathbf{4}$ & Plant height $(\mathrm{cm})$ & 3.89 & 82.75 & 0.05 \\
\hline $\mathbf{5}$ & Primary spike length $(\mathrm{cm})$ & -0.07 & 39.90 & 0.00 \\
\hline $\mathbf{6}$ & Number of spikes/ plant & 0.01 & 0.11 & 0.09 \\
\hline $\mathbf{7}$ & 100 seed weight $(\mathrm{g})$ & 0.43 & 0.40 & 1.08 \\
\hline $\mathbf{8}$ & Seed yield/ plant $(\mathrm{g})$ & 9.92 & 15.68 & 0.63 \\
\hline $\mathbf{9}$ & Oil content $(\%)$ & 0.08 & 0.15 & 0.53 \\
\hline
\end{tabular}


Table.4 General combining ability ( $g c a$ ) effects of parents for nine characters in castor

\begin{tabular}{|c|c|c|c|c|c|c|c|c|c|}
\hline & $\begin{array}{c}\text { Days to } 50 \\
\text { per cent } \\
\text { flowering }\end{array}$ & $\begin{array}{l}\text { Days to } \\
\text { maturity }\end{array}$ & $\begin{array}{l}\text { Nodes up to } \\
\text { primary } \\
\text { spike }\end{array}$ & $\begin{array}{l}\text { Plant } \\
\text { height } \\
(\mathrm{cm})\end{array}$ & $\begin{array}{c}\text { Primary } \\
\text { spike length } \\
(\mathrm{cm})\end{array}$ & $\begin{array}{l}\text { No. of } \\
\text { spikes/ } \\
\text { plant }\end{array}$ & $\begin{array}{c}100 \text { seed } \\
\text { weight }(\mathrm{g})\end{array}$ & $\begin{array}{c}\text { Seed } \\
\text { yield/ } \\
\text { plant (g) }\end{array}$ & $\begin{array}{c}\text { Oil } \\
\text { content } \\
(\%)\end{array}$ \\
\hline \multicolumn{10}{|l|}{ LINES } \\
\hline DPC-9 & $-2.11 * *$ & -0.13 & $-0.65 * *$ & -0.74 & $-3.30 * *$ & $0.24 * *$ & -0.29 & $7.29 * *$ & $0.55 * *$ \\
\hline M-574 & $0.86 * *$ & -0.10 & $0.59 * *$ & $5.44 * *$ & 0.94 & $-0.22 * *$ & 0.08 & -1.81 & -0.34 \\
\hline PPL-18 & $1.26 * *$ & 0.23 & 0.07 & $-4.71 * *$ & $2.36 * *$ & -0.02 & 0.21 & $-5.48 * *$ & -0.21 \\
\hline SE (gi) & 0.30 & 0.29 & 0.11 & 0.74 & 0.62 & 0.07 & 0.22 & 1.24 & 0.17 \\
\hline \multicolumn{10}{|c|}{ TESTERS } \\
\hline PCS-202 & $-1.84 * *$ & $-2.71 * *$ & $-0.90 * *$ & $5.31 * *$ & $2.46^{*}$ & $-0.74 * *$ & $-3.10 * *$ & $-15.37 * *$ & $-1.28 * *$ \\
\hline PCS-225 & $1.60 * *$ & $-2.38 * *$ & 0.11 & $13.51 * *$ & $-4.63 * *$ & $-0.33 *$ & $-3.54 * *$ & $-5.92 *$ & $-1.23 * *$ \\
\hline PCS-124 & $-1.84 * *$ & 0.62 & 0.04 & -0.87 & -0.82 & $1.05^{* *}$ & $2.68 * *$ & $18.63 * *$ & $1.74 * *$ \\
\hline SKI-215 & $2.49 * *$ & $3.07 * *$ & 0.20 & -0.63 & -0.12 & $0.79 * *$ & $3.01 * *$ & $13.30 * *$ & $1.86^{* *}$ \\
\hline PCS-223 & $-1.51 * *$ & $-2.49 * *$ & $-0.59 * *$ & $-12.36 * *$ & $-3.92 * *$ & -0.20 & $-2.43 * *$ & $-9.59 * *$ & $-1.52 * *$ \\
\hline JC-12 & $3.04 * *$ & $4.51 * *$ & $0.42 *$ & $11.86 * *$ & $5.48 * *$ & $0.33^{*}$ & $3.79 * *$ & $9.19 * *$ & $1.03^{* *}$ \\
\hline PCS-136 & $-2.18 * *$ & $-2.27 * *$ & $-0.89 * *$ & $-11.34 * *$ & 2.21 & $-0.65 * *$ & $-1.77 * *$ & $-9.14 * *$ & -0.46 \\
\hline PCS-106 & 0.04 & $1.51 * *$ & -0.33 & $8.88 * *$ & -1.20 & $0.29 *$ & $2.12 * *$ & $10.19 * *$ & 0.50 \\
\hline PCS-171 & $2.27 * *$ & $-1.93 * *$ & $2.49 * *$ & $-8.65 * *$ & $2.32 *$ & $-0.54 * *$ & $-2.66 * *$ & $-18.14^{* *}$ & $-1.05 * *$ \\
\hline DCS-78 & $-2.07 * *$ & $2.07 * *$ & $-0.54 *$ & $-5.72 * *$ & -1.78 & 0.00 & $1.90 * *$ & $6.86 * *$ & 0.41 \\
\hline SE (gj) & 0.55 & 0.54 & 0.21 & 1.35 & 1.14 & 1.33 & 0.41 & 2.26 & 0.31 \\
\hline
\end{tabular}

* Significant at $5 \%$ level, ** Significant at $1 \%$ level 
Table.5 Specific combining ability (sca) effects of parents for nine characters in castor

\begin{tabular}{|c|c|c|c|c|c|c|c|c|c|}
\hline & $\begin{array}{l}\text { Days to } 50 \text { per cent } \\
\text { flowering }\end{array}$ & $\begin{array}{l}\text { Days to } \\
\text { maturity }\end{array}$ & $\begin{array}{l}\text { Nodes up to } \\
\text { primary spike }\end{array}$ & $\begin{array}{l}\text { Plant height } \\
(\mathrm{cm})\end{array}$ & $\begin{array}{l}\text { Primary spike } \\
\text { length }(\mathrm{cm})\end{array}$ & $\begin{array}{l}\text { Spikes/ } \\
\text { plant }\end{array}$ & $\begin{array}{l}100 \text { seed } \\
\text { weight }(\mathrm{g})\end{array}$ & $\begin{array}{l}\text { Seed yield/ } \\
\text { plant }(\mathrm{g})\end{array}$ & $\begin{array}{l}\text { Oil content } \\
(\%)\end{array}$ \\
\hline DPC-9 x PCS-202 & -0.89 & 0.24 & 0.01 & 0.58 & 1.83 & $0.61 *$ & 0.07 & -0.73 & -0.46 \\
\hline DPC-9 x PCS- 225 & $3.67 * *$ & 0.91 & $0.77 *$ & 4.51 & 2.29 & -0.40 & 0.51 & $-8.84 *$ & -0.72 \\
\hline DPC-9 x PCS-124 & $-3.56 * *$ & $-2.09 *$ & 0.47 & $12.69 * *$ & $6.61 * *$ & 0.18 & 0.29 & $8.93 *$ & 0.95 \\
\hline DPC-9 x SKI-215 & -0.56 & -0.53 & 0.08 & $-6.15^{*}$ & $-4.39 *$ & -0.15 & -0.71 & $7.93 *$ & 0.29 \\
\hline DPC-9 x PCS-223 & 0.78 & 1.02 & 0.30 & 3.71 & -1.59 & 0.04 & 0.40 & 1.16 & -0.30 \\
\hline DPC-9 x JC-12 & -0.11 & 0.69 & 0.12 & $-6.64 * *$ & $-4.23 *$ & 0.37 & -0.16 & 0.71 & 0.16 \\
\hline DPC-9 x PCS-136 & 1.11 & 0.13 & 0.43 & $-7.24 * *$ & 0.77 & 0.12 & 0.07 & -3.62 & -0.22 \\
\hline DPC-9 x PCS-106 & -1.78 & -1.31 & -0.20 & -3.66 & 1.69 & -0.32 & 0.51 & 0.71 & 0.22 \\
\hline DPC-9 x PCS-171 & -1.67 & 0.47 & $-2.65 * *$ & $6.40 * *$ & 1.06 & 0.27 & -0.71 & -6.62 & 0.50 \\
\hline DPC-9 x DCS-78 & $3.00 * *$ & 0.47 & 0.68 & -4.20 & $-4.04 *$ & $-0.73 * *$ & -0.27 & 0.38 & -0.42 \\
\hline M-574 x PCS-202 & -0.86 & -0.46 & -0.57 & $-19.07 * *$ & $-9.37 * *$ & -0.04 & 0.37 & 4.37 & 0.80 \\
\hline M-574 x PCS-225 & 0.03 & -0.46 & -0.51 & 0.00 & 2.42 & 0.02 & 0.81 & 6.26 & 0.64 \\
\hline M-574 x PCS-124 & $4.14 * *$ & 0.54 & -0.08 & $-6.89 * *$ & 1.84 & -0.13 & 0.26 & -7.63 & 0.24 \\
\hline M-574 x SKI-215 & 1.14 & 0.10 & -0.17 & $10.07 * *$ & 2.34 & 0.04 & 0.92 & -5.30 & -0.42 \\
\hline M-574 x PCS-223 & -0.52 & -1.01 & -0.44 & -2.20 & $-6.13 * *$ & 0.00 & 0.03 & 2.26 & 0.10 \\
\hline M-574 x JC-12 & 1.59 & -0.34 & -0.06 & 0.04 & 0.44 & -0.07 & -0.19 & 1.81 & -0.15 \\
\hline M-574 x PCS-136 & 0.48 & -0.90 & -0.21 & $6.38 * *$ & 0.54 & -0.26 & -1.30 & -2.86 & 0.01 \\
\hline M-574 x PCS-106 & 0.59 & $1.99 *$ & -0.31 & $6.22 *$ & $-6.98 * *$ & -0.23 & 0.14 & -1.19 & $-1.15^{*}$ \\
\hline M-574 x PCS-171 & $-7.63 * *$ & 0.43 & $2.01 * *$ & -3.58 & 3.16 & 0.03 & -1.41 & 2.14 & 0.06 \\
\hline M-574 x DCS-78 & 1.03 & 0.10 & 0.33 & $9.02 * *$ & $11.73 * *$ & $0.63 * *$ & 0.37 & 0.14 & -0.13 \\
\hline PPL-18 x PCS-202 & 1.74 & 0.21 & 0.56 & $18.49 * *$ & $7.54 * *$ & $-0.57 *$ & -0.43 & -3.63 & -0.33 \\
\hline PPL-18 x PCS-225 & $-3.70 * *$ & -0.46 & -0.25 & -4.51 & $-4.70^{*}$ & 0.39 & -1.32 & 2.59 & 0.08 \\
\hline PPL-18 x PCS-124 & -0.59 & 1.54 & -0.39 & $-5.80^{*}$ & $-8.45 * *$ & -0.06 & -0.54 & -1.30 & $-1.19 *$ \\
\hline PPL-18 x SKI-215 & -0.59 & 0.43 & 0.09 & -3.91 & 2.05 & 0.11 & -0.21 & -2.63 & 0.12 \\
\hline PPL-18 x PCS-223 & -0.26 & -0.01 & 0.15 & -1.51 & $7.72 * *$ & -0.04 & -0.43 & -3.41 & 0.20 \\
\hline PPL-18 x JC-12 & -1.48 & -0.34 & -0.07 & $6.60 * *$ & 3.78 & -0.30 & 0.34 & -2.52 & -0.01 \\
\hline PPL-18 x PCS-136 & -1.59 & 0.77 & -0.22 & 0.86 & -1.32 & 0.14 & 1.23 & 6.48 & 0.21 \\
\hline PPL-18 x PCS-106 & 1.19 & -0.68 & 0.51 & -2.56 & $5.30 * *$ & $0.54 *$ & -0.66 & 0.48 & 0.92 \\
\hline PPL-18 x PCS-171 & $9.30 * *$ & -0.90 & 0.63 & -2.82 & $-4.23 *$ & -0.30 & $2.12 * *$ & 4.48 & -0.56 \\
\hline PPL-18 x DCS-78 & $-4.03 * *$ & -0.57 & $-1.01 * *$ & $-4.82 *$ & $-7.69 * *$ & 0.10 & -0.10 & -0.52 & 0.55 \\
\hline SE & 0.95 & 0.93 & 0.36 & 2.34 & 1.97 & 0.23 & 0.71 & 3.90 & 0.54 \\
\hline
\end{tabular}


Table.6 Performance of four superior crosses for seed yield per plant in castor

\begin{tabular}{|l|c|c|c|c|c|c|c|}
\hline & $\begin{array}{c}\text { Per se } \\
\text { performa } \\
\text { nce }(\mathrm{g})\end{array}$ & $\begin{array}{c}s c a \\
\text { effect }\end{array}$ & \multicolumn{2}{|c|}{ Parent } & \multicolumn{2}{|c|}{ Parent 2} \\
\hline performance & $\begin{array}{c}\text { gca } \\
\text { effect }\end{array}$ & $\begin{array}{c}\text { Per se } \\
\text { performance }\end{array}$ & $\begin{array}{c}\text { gca } \\
\text { effect }\end{array}$ \\
\hline DPC-9 x PCS-124 & 148.33 & $8.93 *$ & 86.00 & $7.29 * *$ & 114.00 & $\begin{array}{c}18.63 * \\
*\end{array}$ \\
\hline DPC-9 x SKI-215 & 142.00 & $7.93 *$ & 86.00 & $7.29 * *$ & 114.67 & $\begin{array}{c}13.30 * \\
*\end{array}$ \\
\hline DPC-9 x PCS-106 & 131.67 & 0.71 & 86.00 & $7.29 * *$ & 118.33 & $\begin{array}{c}10.19 * \\
*\end{array}$ \\
\hline DPC-9 x JC-12 & 130.67 & 0.71 & 86.00 & $7.29 * *$ & 116.33 & $9.19 * *$ \\
\hline
\end{tabular}

Table.7 Contribution of different characters towards total variance

\begin{tabular}{|r|l|c|c|c|}
\hline S.No. & \multicolumn{1}{|c|}{ Character } & Lines & Testers & Line $\mathrm{x}$ Tester \\
\hline $\mathbf{1}$ & Days to 50 per cent flowering & 15.24 & 27.90 & 56.86 \\
\hline $\mathbf{2}$ & Days to maturity & 0.38 & 89.83 & 9.79 \\
\hline $\mathbf{3}$ & Nodes up to primary spike & 15.56 & 52.48 & 31.96 \\
\hline $\mathbf{4}$ & Plant height (cm) & 11.45 & 53.81 & 34.74 \\
\hline $\mathbf{5}$ & Primary spike length $(\mathrm{cm})$ & 14.15 & 21.69 & 64.16 \\
\hline $\mathbf{6}$ & Number of spikes / plant & 7.50 & 71.15 & 21.35 \\
\hline $\mathbf{7}$ & 100 seed weight (g) & 0.54 & 92.82 & 6.64 \\
\hline $\mathbf{8}$ & Seed yield/ plant (g) & 14.38 & 76.42 & 9.20 \\
\hline $\mathbf{9}$ & Oil content (\%) & 8.21 & 77.57 & 14.22 \\
\hline
\end{tabular}

In the present investigation, most of the crosses showing high $s c a$ effects for different characters involved at least one good general combiner for that trait (Table 6). This suggests that either additive $\mathrm{x}$ additive and/or additive $\mathrm{x}$ dominance genetic interaction was predominant in the material under study. In such cases where non-additive gene effects play an important role in association with additive components, recurrent selection or reciprocal recurrent selection approaches can be used to exploit both the components simultaneously.

The per cent contribution of testers was greater than the individual contribution of lines and line $\mathrm{x}$ tester interaction for the traits days to maturity, number of nodes up to primary spike, plant height, number of spikes per plant, 100 seed weight, seed yield per plant and oil content. The contribution of line $\mathrm{x}$ tester interaction was greater than the individual contribution of lines and testers for days to fifty per cent flowering and primary spike length (Table 7).

To synthesize a dynamic population with most of the favorable genes accumulated, it is pertinent to make use of the aforesaid parents, which are good general combiners for several characters, in multiple crossing programmes. Apart from conventional breeding methods relying solely upon additive or additive $\mathrm{x}$ additive type of gene action, population 
improvement appears to be a promising alternative.

Normally, sca effects do not contribute tangibly to the improvement of self-fertilizing crops, except where commercial exploitation of heterosis is feasible. The sca represents the dominance and epistatic interactions that can be related to heterosis. However, in selfpollinated crops like barley, the additive $\mathrm{x}$ additive type of interaction component is fixable in later generations. A breeder's interest, therefore, vests in obtaining transgressive segregants through crosses and producing more potent homozygous lines. Jinks and Jones (1958) emphasized that the superiority of the hybrids might not indicate their ability to yield transgressive segregants; rather, the sca would provide satisfactory criteria.

\section{References}

Aher, A.R., Patel, K. V., Patel, M. P. and Patel, J.A. 2001. Genetic analysis of seed yield and component characters over environments in castor (Ricinus communis L.). Electr. J. Plant Breed. 6(1): 141- 149.

Allard, R. W. 1960. Principles of Plant Breeding. John Wiley and Sons, Inc., New York. pp. 310-315.

Burton, G. W. and De Vane, E. W. 1953. Estimating heritability in tall Fescue (Fescuta arudinaceae) from replicated clonal material. Agron. J. 45: 478-481.

Chandramohan, Y., Vishnuvardhanreddy, A. and Nageshwarrao, T. 2006. Combining ability in Castor, Ricinus communis L. J. Oilseeds Res. 23(2): 178-183.

Chaudhari, G. and Patel, B.N. 2014. Combining ability and gene action for yield and yield component characters of newly developed castor (Ricinus communis L.) hybrid. International Conference on Multidisciplinary Research and Practice. Vol I, Issue VIII: 497 - 500.

Dabholkar, A.R. 1992. Elements of Biometrical Genetics. Concept Publishing Company, New Delhi. Pp: 187-214.

Falconer, D. S. 1981. Introduction to Quantitative
Genetics. 2nd Edn. ELBS. Longman. Pp.14-169.

Jinks, J. L. and Jones, R. M. 1958. Estimation of the components of heterosis. Genetics. 43:223-234.

Johnson, H.W., Robinson, H. F. and Comstock, R. E. 1955. Estimates of genetic and environmental variability in soybeans. Agron. J. 47: 314-318.

Kasture, A. G., Patel, D. A., Patel, R. K., Salunkhe, M. D. and Patel, V. P. 2014. Genetic analysis for seed yield and its components in Castor (Ricinus communis L.) Trends Biosci. 7 (5): 368-372.

Kempthorne, O. 1957. An introduction to genetic statistics. John Wiley and Sons, Inc: New York.

Lavanya, C., Ramanarao, P. V. and Venkatagopinath, V. 2006. Studies on combining ability and heterosis for seed yield and yield components in castor, Ricinus communis L. hybrids. J. Oilseeds Res. 23(2): 174-177.

Mehta, D. R., 2000. Combining ability analysis for yield and its component characters in castor (Ricinus communis L.). Ind. J. Agric. Res. 34(3): 200-202.

Panse, V. G. and Sukhatme, P. V. 1984. Statistical Methods for Agricultural Workers, Indian Council of Agricultural Research, New Delhi, pp.381.

Patel, D. K., Chaudhari, F. P., Patel, M. S. and Thakker, D.A. 2007. Studies on gene action and its attributing characters in castor (Ricinus communis L.) through line $\times$ tester. In: National seminar on Changing global vegetable oils scenario: Issues and challenges before India, January, 29-31, pp. 51-52.

Patel, P.C., Dadheech, A., Dave, P.B. and Makani, A.Y. 2015. Heterosis and combining ability for yield and yield component characters in castor (Ricinus communis L.). Green Farming. 6(5): 970 - 973.

Patel, K.P., Patel, M.P., Joshi, N.R. and Patel, J.A. 2017. General and specific combining ability for quantitative characters in castor (Ricinus communis L.). Electronic J. Plant Breeding. 8(2): $422-427$.

Patel, J. A., Patel, K. V. and Patel, A. R. 2012. Genetic analysis of seed yield and 
component characters over environments in castor (Ricinus communis L.). Ind. J. Agric. Res. 46 (2): 148-154.

Patel, J. R., Saied, M. P., Patel, C. G., Bhatt, R. K. and Bhatt, J. P. 2010. Genetic variability and correlation studies in Castor (Ricinus communis L.). Int. J. Agric. Sci. 6(1): 19131.

Pawar, R. R and Sakhare, S. B. 2016. Combining ability studies in castor. Bioinfolet. 13: 1 -5 .

Punewar, A.A., Patil, A.S., Nandanwar, H.R., Patel, S.M. and Patel, B.N. 2017. Genetic dissection of heterosis and combining ability in castor (Ricinus communis L.) with Line $\mathrm{x}$ Tester analysis. J. Experimental Biology and Agricultural Sciences. 5(1): $77-86$.

Ramesh, M., Lavanya, C., Brahmeshwararao, M.V. and Meena, H. P. 2012. Genetic variability, heritability and genetic advance for yield and yield components in castor (Ricinus communis L.) genotypes. J. Oilseeds Res. 29(1): 101103.

Ramesh, M., Lavanya, C., Sujatha, M., Sivasankar, A., Aruna Kumari, J. and Meena, H.P. 2013. Heterosis and combining ability for yield and yield component traits of newly developed castor (Ricinus communis L.). Bioscan. 8: 1421 - 1424

Ramu, R., N. Sreedhar, C. Lavanya and Ramesh, T. 2002. Combining ability studies in castor (Ricinus communis L.). J. Oilseeds Res. 19(2): 229-230.

Robinson, H. F., Comstock, R. E. and Harvey, P. H. 1949. Estimation of heritability and degree of dominance in corn. Agron. J. 41: 353-359.

Sharma, Y., S. N. Sharma, Joshi and Sain, R. S.
2002 Combining ability analysis for yield and yield contributing characters in sixrowed barley. SABRAO J. Breed. Genet. 34(2): 55-63

Singh, R.K. and Chaudhary, B. D. 1977. Line $\times$ tester analysis. In: Biometrical Methods in Quantitative Genetic Analysis. Kalyani Publishers, Ludhiana. pp.191-200.

Sivasubramanian, S. and Madhavamenon, P. 1973. Genotypic and phenotypic variability in rice. Madras Agric. J. 60: 1093-1096.

Solanki, S. S. 2006. Studies on combining ability analysis and physiological parameters under rainfed conditions in castor, Ricinus communis L. J. Oilseeds Res. 23(1): 3134.

Solanki, S. S., Deora, V. S. and Singh, D. P. 2004. Combining ability of new castor, Ricinus communis L. pistillate line: MCP-1-1. J. Oilseeds Res. 21(2): 274-276.

Sood, S. and Kumar, N. 2011. Genetic estimates of fruit yield and its component traits in bell pepper (Capsicum annuum L. var grossum sendt.). SABRAO J. Breed. Genet. 43 (2): 122-129.

Thakker, D.A., Jadon, B.S., Patel, K. M. and Patel, C. J. 2005. Combining ability over environments in castor, Ricinus communis L. J. Oilseeds Res. 22(2): 321-323.

Vaghela, P.O., Thakkar, D.A., Bhadauria, H.S., Sutariya, D.A., Parmar, S.K. and Prajapati, D.V. 2011. Heterosis and combining ability for yield and its component traits in Indian mustard [Brassica juncea (L.)]. 2(1): 39 - 43

Venkataramana, P., Lavanya, C. and Ratnasree, P. 2005. Combining ability and heterosis studies under rainfed conditions in castor (Ricinus communis L.). Ind. J. Genet. 65(4): 325-326.

\section{How to cite this article:}

Bindu Priya, P., M.V. Nagesh Kumar, V. Gouri Shankar, G. Seshu, G. Anuradha and Venkata Ramana, M. 2018. Studies on Magnitude of Genetic Variability and Estimates of Combining Ability for Yield and its Contributing Traits among Improved Lines of Castor (Ricinus communis L.). Int.J.Curr.Microbiol.App.Sci. 7(01): 3031-3041.

doi: https://doi.org/10.20546/ijcmas.2018.701.360 